Die Verantwortung für eine rezeptpflichtige medikamentöse Therapie liegt immer beim Arzt.

Der Artikel beleuchtet das Vorgehen der praktizierenden Ärzteschaft beim Verschreiben von Medikamenten oder bei der ärztlichen Medikamentenabgabe. Tatsache ist, dass immer mehr Patienten nach Konsultation des Internets mit vorgefassten Meinungen oder selbstgestellten $\mathrm{Di}$ agnosen in die Praxis kommen. In der Regel führen dann das Gespräch und die körperliche Untersuchung zu einer Arbeitshypothese/Diagnose und zur anschliessenden Therapie. Kenne ich einen $\mathrm{Pa}$ tienten und seine Krankengeschichte sehr gut, so kann ich ihm - aufgrund der geschilderten Sachlage - telefonisch sowohl zu Hause als auch über die Pflegefachfrau im Heim ein Medikament zur Einnahme oder zur Abgabe empfehlen. Voraussetzung ist in jedem Fall, dass ich den Patienten physisch kenne. Nur so kann ich die Verantwortung für die Therapie übernehmen.

Dr. med. Ernst Gähler,

Vizepräsident der FMH, Verantwortlicher Ressort Paramedizinische Berufe

\title{
Das ärztliche Rezept im digitalen Zeitalter
}

Andreas Wildi, Max Giger

Korrespondenz:

Dr. med. lic. iur. HSG

Andreas Wildi

Meyerlustenberger Lachenal

Rechtsanwälte

Forchstrasse 452

CH-8032 Zürich

andreas.wildi[at]mll-legal.com

\section{Einführung}

Die ärztliche Tätigkeit folgt dem Algorithmus Anamnese, klinischer Status, Spezialuntersuchungen, Diagnose, Therapieempfehlung, therapeutische Vereinbarung mit dem orientierten Patienten, Durchführung der Therapie, Kontrolle des Therapieerfolgs und allfällige Neueinleitung dieses Algorithmus.

Zunehmend ändert sich in jüngerer Zeit diese uralte Kaskade. Der Patient kennt die Diagnose oder meint, sie zu kennen. Seiner Meinung nach trennt ihn oft nur noch ein Formalismus von der gewünschten Therapie: das Rezept. Er möchte möglichst ohne vom Arzt durchgeführte Anamnese, ohne Erhebung des klinischen Status und ohne Durchführung allfälliger Zusatzuntersuchungen direkt für die selbstindizierte Therapie ein ärztlich ausgestelltes Rezept erhalten. Dieses ermöglicht einerseits den Bezug von Arzneimitteln in einer Apotheke und berechtigt andererseits zur Kostenübernahme der in der Spezialitätenliste (SL) des Bundesamtes für Gesundheit (BAG) aufgeführten Arzneimittel durch die obligatorische Krankenpflegeversicherung (OKP).

Die Patienten versuchen zunehmend selbst zu diagnostizieren aufgrund von Recherchen im Internet oder in Analogie zu früheren ärztlichen Diagnosen ihrer Beschwerden. Und sie wollen die Therapie selbst durchführen. Weshalb soll der Patient zum Arzt, um sich zum x-ten Mal in zehn Jahren gegen die bekannten Migränekopfschmerzen ein Rezept für das immer gleiche Arzneimittel ausstellen zu lassen. Und weshalb soll der Patient der Versandapotheke für NeoCitran ${ }^{\circledast}$ ein Rezept einschicken, während er das Präparat in einer Offizinapotheke von einer Pharmaassistentin ohne weiteres ausgehändigt erhält?

Die ärztliche Kompetenz und die ärztliche Autorität haben demgegenüber auch in einer Internetaufgeklärten Zeit ihre mehrschichtige Berechtigung. Der Arzt soll einerseits Ziele des Heilmittelgesetzes garantieren, nämlich den effektiven und sicheren Einsatz der Heilmittel bzw. den Schutz des Einzelnen

\section{L'ordonnance médicale à l'ère}

\section{du numérique}

En principe, un médecin rédige une ordonnance uniquement pour des patients qu'il a lui-même examinés et lorsque le diagnostic lui est connu. Or les technologies de l'information et de la communication modernes voient l'émergence de nouvelles formes de prescription, de plus en plus d'ordonnances étant établies sans que le médecin n'ait vu le patient au préalable. Dans la plupart des cas, ces nouvelles formes de prescription sans contact physique entre le médecin et son patient sont légales, mais l'établissement d'ordonnances a posteriori n'est pas sans poser problème. L'établissement d'ordonnances sans consultation médicale préalable pour des médicaments remis par correspondance par exemple va même à l'encontre des principes éthiques et juridiques de notre profession.

vor den Gefahren einer unbedachten Arzneimittelanwendung. Andererseits soll der Arzt gemäss Krankenversicherungsgesetz garantieren, dass in der SL aufgeführte Arzneimittel zweckmässig und wirtschaftlich zu Lasten der Solidargemeinschaft der Versicherten angewandt werden.

Hierzu hat der Arzt den Patienten persönlich zu beraten und zu behandeln.

\section{Sachverhalt}

Vor diesem Hintergrund stellt sich die Frage, wie viel Entscheidungsautorität und -autonomie beim Arzt verbleiben müssen. Wie verhält er sich berufsethisch 
und rechtlich korrekt, wenn sein althergebrachter Tätigkeitsablauf durch moderne Interaktionstools Modifizierungen erfährt?

Neben offensichtlich missbräuchlichen Zeugnisforderungen, die keiner Erläuterung bedürfen, werfen insbesondere folgende Konstellationen immer wieder Fragen auf:

a) Der aus der Sprechstunde bekannte Patient bittet um telefonische Konsultation und Rezeptausstellung wegen rezidivierender Migräne.

b) Der Patient lässt sich in der Offizin das vom Arzt wiederholt verschriebene Arzneimittel der Abgabekategorie A oder $\mathrm{B}$ aushändigen und bittet die Apothekerin darum, sie solle selbst mit dem Arzt die notwendigen Formalitäten regeln.

c) Die Pflegefachfrau des Pflegeheims wünscht entweder ex ante oder ex post die Verschreibung eines Arzneimittels für einen dem Arzt persönlich bekannten Patienten, ohne dass der Arzt den Patienten im Hinblick auf diese spezifische Verschreibung persönlich untersucht bzw. untersuchte.

d) Die Versandapotheke verlangt eine ärztliche Verschreibung für eine Bestellung eines ihr über einen Fragebogen «bekannten» Patienten/Kunden, mit dem weder die verantwortliche Apothekerin noch der um das Rezept gebetene Arzt persönlichen Kontakt hatten.

e) Der online und/oder telefonisch individuell ärztlich beratene Patient verlangt vom konsultierten Arzt des Beratungsdienstes ein Rezept.

\section{Rechtliches}

\section{Sachverhalte a) und e)}

Diese Konstellationen redimensionieren den erwähnten ärztlichen Vorgehensalgorithmus, sie tasten ihn aber nicht verfälschend an. Es soll dem Arzt möglich sein, sein korrektes Vorgehen entweder detaillierter oder kursorischer zu handhaben. Der Arzt entscheidet am Telefon, ob ihm die Angaben für eine Arbeitsdiagnose genügen. Er trägt bewusst die Verantwortung für die darauf basierenden therapeutischen Entscheide oder für den Rat zur physischen Kontaktaufnahme mit einem weiteren Arzt, um die Diagnose zu sichern. Aus grundsätzlicher rechtlicher Sicht ist damit gegen die Praxis der Rezeptur via Medienmittel beim bekannten Patienten nichts einzuwenden. Betäubungsmittel sind davon allerdings ausgeschlossen. Betäubungsmittel dürfen nur von Ärzten mit kantonaler Bewilligung zur selbständigen Berufsausübung auf dem offiziellen amtlichen Formular einzig für Patienten, die sie selber untersucht haben, verschrieben werden (Art. 9 BetmG in Verbindung mit Art. 43 Abs. 1 BetmV). Einige Kantone verlangen allerdings, dass der Patient dem Arzt in jedem Fall persönlich bekannt sein muss. Die Gerichte waren bis dato aber noch nicht mit der Frage konfrontiert, ob damit «von Angesicht zu Angesicht im selben nicht-digitalen Raum» gemeint sein könnte.

\section{Sachverhalt b)}

Wenn eine Apothekerin ein Arzneimittel der Abgabekategorie A oder B ohne Rezept aushändigt, trägt sie die vollumfängliche Verantwortung. Das Ex-post-Einholen eines Rezeptes bei einem Arzt entbindet sie nicht davon. Der Arzt ist nicht zur Ausstellung des Rezepts verpflichtet. Er wird dies - sofern er die Aushändigung des Arzneimittels bzw. die Therapie für korrekt einstuft - für einen ihm gutbekannten Patienten meistens durchführen. Für einen ihm nicht bekannten Patienten ist die Rezeptierung problematisch, sowohl aus heilmittel- wie auch aus sozialversicherungsrechtlicher Sicht.

Sie lässt sich mit einer sorgfältigen Auftragserfüllung im Prinzip nicht vereinbaren, weil so die Rezeptierung seitens des damit grundsätzlich betrauten Arztes die entscheidenden Schritte zu seiner Diagnosestellung nicht mitumfasst und daher die optimale Therapie, allenfalls ohne Arzneimittel, nicht sichergestellt ist. Gleichzeitig darf in einem Dreiervertrauensverhältnis zwischen Arzt, Apothekerin und Patientin in vielen Fällen dennoch von einer korrekten Arzneimittelabgabe ausgegangen werden, weil sich aus der konkreten Alltagssituation heraus ergibt, dass die Therapie optimal gewählt worden war. Nicht legal sind Lösungen, bei denen ein Arzt einem Apotheker, ohne dass der Arzt dessen Kunden/Patienten kennt, systematisch ex post für alle schon abgegebenen rezeptpflichtigen Arzneimittel ein Rezept ausstellt, die der Apotheker ohne Ex-ante-Rezept abgegeben hat.

In Zukunft sollen Apothekerinnen einige Arzneimittel der heutigen Abgabekategorie B selbständig ohne ärztliche Verschreibung anwenden dürfen (vgl. laufende HMG-Revision in den eidgenössischen Rä-

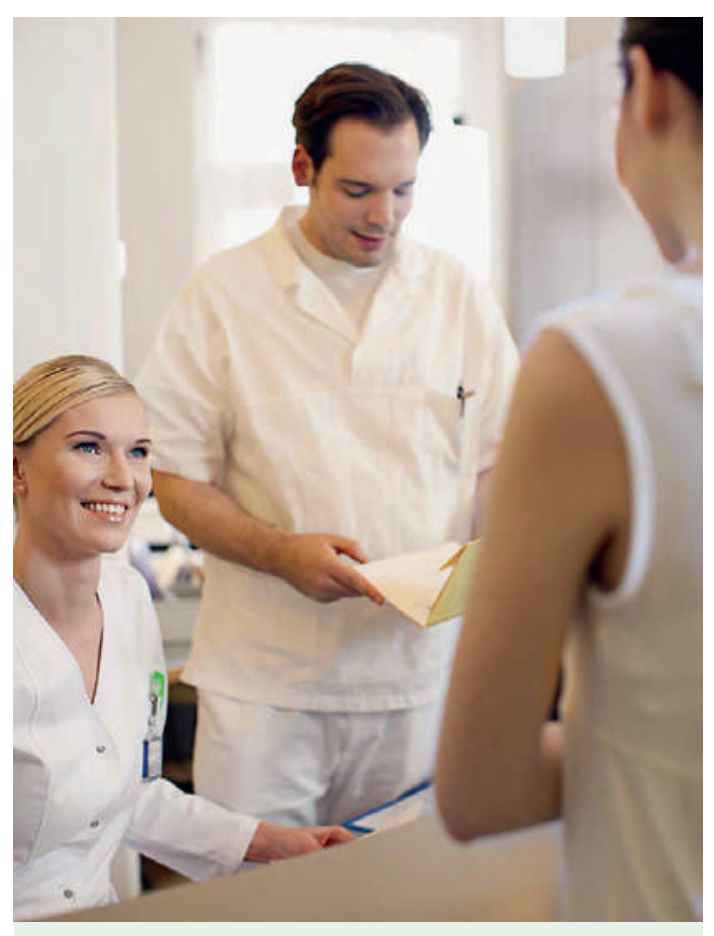

Nur wer einen Patienten persönlich kennt, kann die Verantwortung für seine Therapie übernehmen. 
ten). Es ist zu begrüssen, dass althergebrachte Schranken zwischen ärztlicher und pharmazeutischer Tätigkeit zugunsten einer zeitgemässen patientenorientierten Heilmittelanwendung abgebaut werden. Um dem erwähnten Algorithmus, der unabhängig vom Handelnden gelten muss, aber in jedem Fall Sorge zu tragen, wollen die Details wohlbedacht sein. An dieser Stelle sei aber diesbezüglich nicht vorgegriffen.

\section{Sachverhalt c)}

Dieser vermeintlich gegenüber b) identische Fall dient vielfach als Generallegitimation für Rezepte ausserhalb des Standardvorgehens. Man hat es immer schon so gemacht. Die Ärzte kennen dies aus der Arbeit während der Weiterbildung und Tätigkeit als angestellte Fachärzte in stationären und ambulanten Institutionen oder später aus ihrer selbständigen Tätigkeit als Heimarzt.
Gesundheitswesen nicht selbst bedienen darf, sondern fachlich unterstützt über seine Therapie mitentscheiden soll, dann ist der Versandhandel eines Arzneimittels ohne Ex-ante-Rezept als widerrechtlich abzulehnen. Ein Ex-post-Rezept heilt die fehlende Beratung durch die Apothekerin nicht, die der Patient im Sachverhalt b immerhin erhält. Dabei geht es beim Versandhandelsrezept nicht um Abgrenzungsfragen zwischen Arzt und Apothekerin, sondern um die Unmöglichkeit, allein mittels elektronischen Fragebogens den Gesundheitszustand des Patienten ausreichend einzuschätzen, um eine Arbeitsdiagnose zu stellen, geschweige denn die Indikation für eine Arzneimitteltherapie zu stellen. Der eingangs erwähnte Algorithmus ist verletzt.

Der Arzt kennt beim Ex-post-Rezeptieren einzig die Daten des Fragebogens und das von der Apothekerin abgegebene Arzneimittel. Aufgrund dieser medizi-

\section{«Der Patient kennt die Diagnose oder meint, sie zu kennen.»}

Dieser Fall gleicht dem Ex-post-Rezeptieren via Apotheke, er ist aber - und das ist entscheidend nicht derselbe. In der Apotheke äussert der Kunde einen expliziten Therapiewunsch, der Apotheker erfüllt ihn und lässt zwecks Kostenübernahme durch die OKP und allenfalls zur persönlichen vermeintlichen Absicherung die Abgabe des Arzneimittels hinterher durch ein Rezept legitimieren.

Im Pflegeheim hingegen klagt der Patient über Schmerzen und ersucht das Pflegepersonal um geeignete Abhilfe. Diese ist im Wesentlichen bereits vorgezeichnet, die standardisierten «Schmerzreserven» sind vom verantwortlichen Arzt festgelegt worden und sind der Pflegefachperson bekannt. Und der Lackmustest, ob rechtlich zulässig oder nicht, liegt genau in dieser explizit formulierten - oder zumindest möglichen Vordefinition: Ein Arzt kann mit der Pflegefachperson mehr oder weniger denkbare Situationen durchspielen und vorsorglich das Vorgehen festlegen sowie enger oder weiter gefasste Rezepte erstellen. Er kann z. B. anordnen, unverzüglich eine Therapie mit einem definierten Antibiotikum zu beginnen, sollten im Voraus bestimmte Bedingungen z. B. Dysurie begleitet von Fieber und positivem Urinschnelltest gegeben sein. Hier sind kreative und je nach Patient und Kompetenz der Pflegeperson individuelle Lösungen möglich. Im Jahresdauerrezept für chronisch Kranke findet sich der gleiche, legitime Ansatz: Die Bedingungen für die Erstellung des Rezepts sind im Voraus definiert.

\section{Sachverhalt d)}

Die Antwort auf die in letzter Zeit vielerorts diskutierte Frage leitet sich aus dem bereits Gesagten ab. Wenn sichergestellt werden soll, dass sich in der Schweiz ein Kunde / Patient / mündiger Bürger im nisch ungenügenden Angaben dürfte er einen Kunstfehler begehen, wenn er diese Bestellung mittels Expost-Rezept gutheisst.

In entsprechenden Beratungen vor der Einführung des Heilmittelgesetzes waren die eidgenössischen Räte denn auch der Ansicht gewesen, jeder Patient solle eine konkrete Beratung erfahren müssen, da es sich effektiv um ein «Beratung-erfahren-Dürfen» zu seinem Wohle handelt. Dementsprechend wurden im Versandhandel nicht nur Arzneimittel der Abgabekategorie A und B einer ärztlichen Verschreibung unterstellt, sondern auch Arzneimittel der Kategorien $\mathrm{C}$ und $\mathrm{D}$.

Des Weiteren hätte der Gesetzgeber den Drogisten die Erlaubnis zum Versandhandel von Arzneimitteln der Abgabekategorie D ebenfalls einräumen müssen, hätte er eine Ex-post-Kontrolle als genügend angesehen.

\section{Zukünftiges}

Die laufende HMG-Revision könnte zur Präzisierung des Geschilderten genutzt werden. Dabei ist insbesondere auf Kohärenz der Regeln zu achten. Durchaus sollen moderne Interaktionsformen zwischen Kunde/ Patient und Fachperson rechtlich nicht behindert werden.

Es sollen aber die neuen, oft digital unterstützten Wege wirksamen Checks \& Balances unterstellt sein. Dabei sollen alle Fachpersonen entsprechend ihrer fachspezifischen Kompetenzen Arzneimittel verordnen und abgeben dürfen. Gleichzeitig müssen die möglichen Konstellationen der ineinandergreifenden Handlungen die Aufgaben, die Verantwortlichkeiten und die Qualitätskontrollen pro mögliche Konstellation klar zuweisen. Andernfalls leidet der effektive und sichere Arzneimitteleinsatz. 